\title{
Chebyshev Wavelet collocation method for solving a class of linear and nonlinear nonlocal boundary value problems
}

\author{
İbrahim Çelik ${ }^{\mathrm{a}^{*}}$ \\ ${ }^{a}$ Department of Mathematics, Faculty of Science and Arts, Pamukkale University, Denizli, Turkey \\ ${ }^{*}$ Corresponding author E-mail: i.celik@pau.edu.tr
}

\section{Article Info}

Keywords: Approximate solution, Boundary-value problems, Chebyshev Wavelet, Colocation method, Nonlocal boundary conditions 2010 AMS: 34B10, 65D20, 65T60

Received: 8 May 2018

Accepted: 21 June 2018

Available online: 30 June 2018

\begin{abstract}
This study proposes the Chebyshev Wavelet Colocation method for solving a class of rthorder Boundary-Value Problems (BVPs) with nonlocal boundary conditions. This method is an extension of the Chebyshev wavelet method to the linear and nonlinear BVPs with a class of nonlocal boundary conditions. In this study, the method is tested on second and fourth-order BVPs and approximate solutions are compared with the existing methods in the literature and analytical solutions. The proposed method has promising results in terms of the accuracy.
\end{abstract}

\section{Introduction}

Many physical phenomena may be modelled by differential equations with nonlocal boundary conditions. Therefore, they have a great attention for researchers of mathematics and physics. Nonlocal conditions occur when values of a function on the boundary are depended on values inside the domain or when direct measurements on the boundary are not taken. These problems with nonlocal boundary conditions are found in many problems such as population dynamics, the process of heat conduction, control theory, theory of elastic stability, evolution equation for species population densities, image processing, porous media flow and turbulence [1,2]. Henderson et al[3] considered uniqueness questions for certain nonlocal boundary value problems for the nth-order linear differential equation. Xue [4] studied the existence of integral solutions for nonlinear differential equations with nonlocal initial conditions in Banach spaces. Babak [5] investigated the uniqueness and existence of nonlocal initial problems for a system of nonlinear parabolic equations weakly coupled with ordinary differential equations. Liang et al [6] established some new theorems about the existence and uniqueness of solutions for semilinear integrodifferential equations with nonlocal initial conditions. Geng et al [7] gave an effective method for solving nonlocal fractional boundary value problems based on the reproducing kernel theory. Zhou et al [8] discussed the nonlocal Cauchy problem for the fractional evolution equations. All methods given here such as, Finite Difference Method (FDM) [9], Shooting Method [10, 11], Adomian Decomposition Method (ADM) [12], Variational Iteration Method (VIM) [13], Homotopy Analysis Method (HAM) [14], Sinc-Collocation Method (SCM) [15], Differential Transform Method (DTM) [16], Optimal Homotopy Asymptotic Method (OHAM) [17], combination of the VIM and the Homotopy Perturbed Method (HPM) [18], Reproducing Kernel Method (RKM) [19, 20], Monotone Iterative [21] and a spectral method based on operational matrices of Bernstein polynomials using collocation method [22] were used to solve multi-point BVPs. Tzanetis et al [23] studied a nonlocal problem modelling Ohmic heating with variable thermal conductivity including an analysis of the asymptotic behaviour and the blow-up of solutions. Bogoya et al [24] studied a nonlocal diffusion model analogous to heat equation with Neumann boundary conditions and proved an existence and uniqueness of solutions. Pao [25] studied some dynamical property of a reaction-diffusion equation with nonlocal boundary condition. Pao et al $[26,27]$ investigated a class of fourth-order nonlinear and semilinear elliptic boundary value problem with nonlocal boundary condition.

The Legendre and Chebyshev wavelets operational matrixes of integration and product operation matrix have been introduced in [28, 29, 30, 31]. Our analyses show that there are some disadvantages in applying Legendre wavelet and Chebyshev wavelet. In[32, 33], these disadvantages are eliminated by Çelik with the Chebyshev Wavelet Collocation Method.

This study presents a Chebyshev Wavelet Collocation Method for the solution of the rth-order linear and nonlinear BVPs given in the following form: 


$$
\begin{gathered}
y^{(r)}(x)=\sum_{i=1}^{r} A_{i}(x) \frac{d^{r-i} y(x)}{d x^{r-i}}+g(x) \\
y^{(r)}(x)=F\left(x, y(x), y^{\prime}(x), \ldots, y^{(r-1)}(x)\right)
\end{gathered}
$$

with the nonlocal boundary conditions

$$
\left\{\begin{array}{l}
y^{(i-1)}\left(x_{j}\right)=b_{i, j}, 1 \leq i \leq m_{j}, 1 \leq j \leq \beta \\
y\left(x_{\beta+1}\right)-y\left(x_{\beta+2}\right)=b_{r}
\end{array}\right.
$$

where $m_{1}, m_{2}, \ldots, m_{\beta}$ are positive integers satisfied $m_{1}+m_{2}+\ldots+m_{\beta}=r-1$ and $a<x_{1}<x_{2}<\ldots<x_{\beta+2}<b, b_{i, j}, b_{r}$ are real numbers. The uniqueness of the BVP in Eqs. (1.1), (1.3) has been discussed in [4].

Chebyshev wavelet collocation method is based on the approximation by the truncated Chebyshev wavelets series. By using the Chebyshev collocation points, algebraic equation system has been obtained. The coefficients of the Chebyshev wavelet series can be found from the solution of the algebraic equation system. The method is applied to the linear and nonlinear boundary value problems with nonlocal boundary conditions. Calculations demonstrated that the accuracy of the Chebyshev wavelet collocation method is quite good even for the case of a small number of grid points.

\section{Chebyshev Wavelet method}

Wavelets have been used in many different fields of science and engineering in recent years. They constitute a family of functions constructed from dilation and translation of a single function called the mother wavelet. If the dilation parameter $a$ and the translation parameter $b$ vary continuously, the following family of continuous wavelets can be obtained [34]

$$
\psi_{a, b}(x)=|a|^{1 / 2} \psi\left(\frac{x-b}{a}\right), a, b \in R, a \neq 0 .
$$

Chebyshev wavelets are written as

$$
\psi_{n m}(x)=\psi(k, n, m, x)
$$

where $k=0,1,2, \ldots, n=1,2, \ldots, 2^{k}, m$ is degree of Chebyshev polynomials of the first kind and $x$ denotes the normalized time. They are defined on the interval $[0,1)$ by:

$$
\psi_{n m}(x)=\left\{\begin{array}{l}
\frac{\alpha_{m} 2^{k / 2}}{\sqrt{\pi}} T_{m}\left(2^{k+1} x-2 n+1\right), \quad \frac{n-1}{2^{k}} \leq x<\frac{n}{2^{k}} \\
0 \quad \text { otherwise }
\end{array}\right.
$$

where

$$
\alpha_{m}= \begin{cases}\sqrt{2} & m=0 \\ 2 & m=1,2, \ldots\end{cases}
$$

and $T_{m}\left(2^{k+1} x-2 n+1\right)$ are Chebyshev polynomials of the first kind of degree $m$ orthogonal with respect to the weight function $w_{n}(x)=$ $w\left(2^{k+1} x-2 n+1\right)=\frac{1}{\sqrt{1-\left(2^{k+1} x-2 n+1\right)^{2}}}$ on $[-1,1][35]$.

A function $f(x) \in L_{w}^{2}[0,1]$ may be expanded as:

$$
f(x)=\sum_{n=1}^{\infty} \sum_{m=0}^{\infty} f_{n m} \psi_{n m}(x)
$$

where

$$
f_{n m}=\left\langle f(x), \psi_{n m}(x)\right\rangle
$$

and $\langle.,$.$\rangle denotes the inner product with weight function w_{n}(x)$ in Eq. (2.4).

Truncated form of Eq. (2.3) can be written as:

$$
f(x) \cong \sum_{n=1}^{2^{k}} \sum_{m=0}^{M-1} f_{n m} \psi_{n m}(x)=C^{T} \Psi(x)
$$

where $C$ and $\Psi(x)$ are $2^{k} M \times 1$ columns vectors given by:

$$
\begin{gathered}
C^{T}=\left[f_{10}, f_{11}, \ldots, f_{1 M-1}, f_{20}, \ldots, f_{2 M-1}, \ldots, f_{2^{k} 0}, \ldots, f_{2^{k} M-1}\right] \\
\Psi(x)=\left[\psi_{10}, \psi_{11}, \ldots, \psi_{1 M-1}, \psi_{20}, \ldots, \psi_{2 M-1}, \ldots, \psi_{2^{k} 0}, \ldots, \psi_{2^{k} M-1}\right]^{T}
\end{gathered}
$$

The integration of the $\psi_{n m}(x)$ given in Eq. (2.2) can be represented as

$$
p_{n m}(x)=\int_{0}^{x} \psi_{n m}(s) d s
$$


For $m=0, m=1$ and $m>1, p_{n m}(x)$ can be obtained as

$$
\begin{aligned}
& p_{n 0}(x)= \begin{cases}0 & \begin{array}{l}
0 \\
\frac{\alpha_{0} 2^{-k / 2-1}}{\sqrt{\pi}}\left[T_{1}\left(2^{k+1} x-2 n+1\right)+T_{0}\left(2^{k+1} x-2 n+1\right)\right]
\end{array} \\
\frac{\alpha_{0} 2^{-k / 2}}{\sqrt{\pi}} T_{0}\left(2^{k+1} x-2 n+1\right) & \frac{n-1}{2^{k}} \leq x<\frac{n-1}{2^{k}} \\
2^{k} & \frac{n}{2^{k}} \leq x<1\end{cases} \\
& p_{n 1}(x)= \begin{cases}0 & 0 \leq x<\frac{n-1}{2^{k}} \\
\frac{\alpha_{1} 2^{-k / 2-3}}{\sqrt{\pi}}\left[T_{2}\left(2^{k+1} x-2 n+1\right)-T_{0}\left(2^{k+1} x-2 n+1\right)\right] & \frac{n-1}{2^{k}} \leq x<\frac{n}{2^{k}} \\
0 & \frac{n^{n}}{2^{k}} \leq x<1\end{cases} \\
& p_{n m}(x)= \begin{cases}0 & 0 \leq x<\frac{n-1}{2^{k}} \\
\frac{\alpha_{m} 2^{-k / 2-2}}{\sqrt{\pi}}\left[\frac{T_{m+1}(u)-(-1)^{m+1}}{m+1}-\frac{T_{m-1}(u)-(-1)^{m-1}}{m-1}\right] & \frac{n-1}{2^{k}} \leq x<\frac{n}{2^{k}} \\
\frac{\alpha_{m} 2^{-k / 2-2}}{\sqrt{\pi}}\left[\frac{1-(-1) m+1}{m+1}-\frac{1-(-1)^{m-1}}{m-1}\right] & \frac{n}{2^{k}} \leq x<1\end{cases}
\end{aligned}
$$

where $u=2^{k+1} x-2 n+1$. The integration of the $\Psi(x)$ can be represented as

where

$$
\int_{0}^{x} \Psi(s) d s=\left[p_{10}, p_{11}, \ldots, p_{1 M-1}, p_{20}, \ldots, p_{2 M-1}, \ldots, p_{2^{k}}, \ldots, p_{2^{k} M-1}\right]^{T}=P_{1} \Psi_{1}(x)
$$

$$
\begin{gathered}
\Psi_{1}(x)=\left[\psi_{10}, \psi_{11}, \ldots, \psi_{1 M}, \psi_{20}, \ldots, \psi_{2 M}, \ldots, \psi_{2^{k} 0}, \ldots, \psi_{2^{k} M}\right]^{T} \\
L_{1}=\left[\begin{array}{ccccccccc}
\frac{\sqrt{2}}{2} & 0 & 0 & \cdots & 0 & 0 & 0 & 0 \\
-\frac{\sqrt{2}}{4} & 0 & \frac{1}{4} & 0 & \cdots & 0 & 0 & 0 & 0 \\
-\frac{\sqrt{2}}{3} & -\frac{1}{2} & 0 & \frac{1}{6} & \cdots & 0 & 0 & 0 & 0 \\
\vdots & \vdots & \vdots & \vdots & \ddots & \vdots & \vdots & \vdots & \vdots \\
\frac{\sqrt{2}}{2}\left(\frac{(-1)^{M-3}}{M-3}-\frac{(-1)^{M-1}}{M-1}\right) & 0 & 0 & 0 & \cdots & -\frac{1}{2(M-3)} & 0 & \frac{1}{2(M-1)} & 0 \\
\frac{\sqrt{2}}{2}\left(\frac{(-1)^{M-2}}{M-2}-\frac{(-1)^{M}}{M}\right) & 0 & 0 & 0 & \cdots & 0 & -\frac{1}{2(M-2)} & 0 & \frac{1}{2 M}
\end{array}\right] \\
F_{1}=\left[\begin{array}{cccc}
2 & 0 & \cdots & 0 \\
0 & 0 & \cdots & 0 \\
\frac{2 \sqrt{2}}{3} & 0 & \cdots & 0 \\
\vdots & \vdots & \ddots & \vdots \\
\frac{\sqrt{2}}{2}\left(\frac{1-(-1)^{M-1}}{M-1}-\frac{1-(-1)^{M-3}}{M-3}\right) & 0 & \cdots & 0 \\
\frac{\sqrt{2}}{2}\left(\frac{1-(-1)^{M}}{M}-\frac{1-(-1)^{M-2}}{M-2}\right) & 0 & \cdots & 0
\end{array}\right] P_{1}=\frac{1}{2^{k+1}}\left[\begin{array}{cccccc}
L_{1} & F_{1} & F_{1} & \cdots & F_{1} & F_{1} \\
0 & L_{1} & F_{1} & \cdots & F_{1} & F_{1} \\
\vdots & \vdots & \vdots & \ddots & \vdots \\
0 & 0 & 0 & \cdots & L_{1} & F_{1} \\
0 & 0 & 0 & \cdots & 0 & L_{1}
\end{array}\right]
\end{gathered}
$$

The second integrations of the $\Psi(x)$ can be represented as

$$
\int_{0}^{x} \int_{0}^{x_{1}} \Psi(s) d s d x_{1}=\int_{0}^{x} P_{1} \Psi_{1}\left(x_{1}\right) d x_{1}=P_{1} \int_{0}^{x} \Psi_{1}\left(x_{1}\right) d x_{1}=P_{1} P_{2} \Psi_{2}(x)
$$

The $\mathrm{r}^{t h}$ integrations of the $\Psi(x)$ can be represented as

$$
\int_{0}^{x} \int_{0}^{x_{1}} \int_{0}^{x_{2}} \cdots \int_{0}^{x_{r-1}} \Psi(s) d s d x_{r-1} d x_{r-2} \cdots d x_{1}=P_{1} P_{2} \cdots P_{r} \Psi_{r}(x)
$$

where

$$
L_{r}=\left[\begin{array}{ccccccccccccc}
1 & \frac{\sqrt{2}}{2} & 0 & 0 & \cdots & 0 & 0 & 0 & 0 & \cdots & 0 & 0 & 0 \\
\frac{-\sqrt{2}}{4} & 0 & \frac{1}{4} & 0 & \cdots & 0 & 0 & 0 & 0 & \cdots & 0 & 0 & 0 \\
\frac{-\sqrt{2}}{3} & \frac{-1}{2} & 0 & \frac{1}{6} & \cdots & 0 & 0 & 0 & 0 & \cdots & 0 & 0 & 0 \\
\vdots & \vdots & \vdots & \vdots & \ddots & \vdots & \vdots & \vdots & \vdots & \ddots & \vdots & \vdots & \vdots \\
\frac{\sqrt{2}}{2}\left(\frac{(-1)^{M-3}}{M-3}-\frac{(-1)^{M-1}}{M-1}\right) & 0 & 0 & 0 & \cdots & \frac{-1}{2(M-3)} & 0 & \frac{1}{2(M-1)} & 0 & \cdots & 0 & 0 & 0 \\
\frac{\sqrt{2}}{2}\left(\frac{(-1)^{M-2}}{M-2}-\frac{(-1)^{M}}{M}\right) & 0 & 0 & 0 & \cdots & 0 & \frac{-1}{2(M-2)} & 0 & \frac{1}{2 M} & \cdots & 0 & 0 & 0 \\
\frac{\sqrt{2}}{2}\left(\frac{(-1)^{M-1}}{M-1}-\frac{(-1)^{M+1}}{M+1}\right) & 0 & 0 & 0 & \cdots & 0 & 0 & \frac{-1}{2(M-1)} & 0 & \cdots & 0 & 0 & 0 \\
\vdots & \vdots & \vdots & \vdots & \ddots & \vdots & \vdots & \vdots & \vdots & \ddots & \vdots & \vdots & \vdots \\
\frac{\sqrt{2}}{2}\left(\frac{(-1)^{M-3+r}}{M-3+r}-\frac{(-1)^{M+r-1}}{M+r-1}\right) & 0 & 0 & 0 & \cdots & 0 & 0 & 0 & 0 & \cdots & \frac{-1}{2(M-3+r)} & 0 & \frac{1}{2(M-1+r)}
\end{array}\right]
$$




$$
F_{r}=\left[\begin{array}{cccc}
2 & 0 & \cdots & 0 \\
0 & 0 & \cdots & 0 \\
\frac{2 \sqrt{2}}{3} & 0 & \cdots & 0 \\
\vdots & 0 & \ddots & 0 \\
\frac{\sqrt{2}}{2}\left(\frac{1-(-1)^{M-1}}{M-1}-\frac{1-(-1)^{M-3}}{M-3}\right) & 0 & \cdots & 0 \\
\frac{\sqrt{2}}{2}\left(\frac{1-(-1)^{M}}{M}-\frac{1-(-1)^{M-2}}{M-2}\right) & 0 & \cdots & 0 \\
\frac{\sqrt{2}}{2}\left(\frac{1-(-1)^{M+1}}{M+1}-\frac{1-(-1)^{M-1}}{M-1}\right) & 0 & \cdots & 0 \\
\vdots & 0 & \ddots & 0 \\
\frac{\sqrt{2}}{2}\left(\frac{1-(-1)^{M+r-1}}{M+r-1}-\frac{1-(-1)^{M+r-3}}{M+r-3}\right) & 0 & \cdots & 0
\end{array}\right] P_{r}=\frac{1}{2^{k+1}}\left[\begin{array}{cccccc}
L_{r} & F_{r} & F_{r} & \cdots & F_{r} & F_{r} \\
0 & L_{r} & F_{r} & \cdots & F_{r} & F_{r} \\
\vdots & \vdots & \vdots & \ddots & \vdots & \vdots \\
0 & 0 & 0 & \cdots & L_{r} & F_{r} \\
0 & 0 & 0 & \cdots & 0 & L_{r}
\end{array}\right]
$$

and

$$
\Psi_{r}(x)=\left[\psi_{10}, \psi_{11}, \ldots, \psi_{1 M+r-1}, \psi_{20}, \ldots, \psi_{2 M+r-1}, \ldots, \psi_{2^{k} 0}, \ldots, \psi_{2^{k} M+r-1}\right]^{T}
$$

Dimensions of the matrices $L_{r}$ and $F_{r}$ are $(M+r-1) \times(M+r)$. Hence $P_{r}$ has the dimension $2^{k}(M+r-1) \times 2^{k}(M+r)$.

\section{Chebyshev Wavelet collocation method for BVPs with nonlocal conditions}

Consider Eq. (1.1) or Eq. (1.2) with the nonlocal boundary conditions

$$
\left\{\begin{array}{l}
y^{(i-1)}\left(x_{j}\right)=b_{i, j}, 1 \leq i \leq m_{j}, 1 \leq j \leq \beta \\
y\left(x_{\beta+1}\right)-y\left(x_{\beta+2}\right)=b_{r}
\end{array}\right.
$$

We assume that $y^{(r)}(x)$ can be expanded in terms of truncated Chebyshev wavelet series as

$$
y^{(r)}(x)=\sum_{n=1}^{2^{k}} \sum_{m=0}^{M-1} f_{n m} \psi_{n m}(x)=C^{T} \Psi(x) .
$$

By successively integrating Eq. (3.1) from 0 to $x$, the following equations are obtained

$$
\begin{gathered}
y^{(r-1)}(x)=\int_{0}^{x} C^{T} \Psi(s) d s+y^{(r-1)}(0)=C^{T} P_{1} \Psi_{1}(x)+y^{(r-1)}(0) \\
y^{(r-2)}(x)=C^{T} P_{1} P_{2} \Psi_{2}(x)+x y^{(r-1)}(0)+y^{(r-2)}(0) \\
y^{(r-3)}(x)=C^{T} P_{1} P_{2} P_{3} \Psi_{3}(x)+\frac{x^{2}}{2} y^{(r-1)}(0)+x y^{(r-2)}(0)+y^{(r-3)}(0) \\
y^{\left(m_{j}\right)}(x)=C^{T} P_{1} P_{2} P_{3} \ldots P_{r-m_{j}} \Psi_{r-m_{j}}(x)+\sum_{s=1}^{r-m_{j}} \frac{x^{s-1}}{(s-1) !} y^{\left(s+m_{j}-1\right)}(0)
\end{gathered}
$$

Theorem 3.1. Chebyshev wavelet expression for zth-order derivatives of unknown function $y(x)$ satisfying nonlocal boundary conditions

$$
\left\{\begin{array}{l}
y^{(i-1)}\left(x_{j}\right)=b_{i, j}, 1 \leq i \leq m_{j}, 1 \leq j \leq \beta \\
y\left(x_{\beta+1}\right)-y\left(x_{\beta+2}\right)=b_{r}
\end{array}\right.
$$

are given as for $z=0,1, \ldots, m_{j}-1$ :

$$
\begin{aligned}
y^{(z)}(x) & =C^{T}\left(P_{1} P_{2} \ldots P_{r-z} \Psi_{r-z}(x)-\sum_{h=1}^{m_{j}-z} \frac{\left(x-x_{j}\right)^{h-1}}{(h-1) !} P_{1} P_{2} \ldots P_{r+1-z-h} \Psi_{r+1-z-h}\left(x_{j}\right)\right) \\
+ & \sum_{s=2}^{r-m_{j}}\left(\frac{x^{s-1+m_{j}-z}}{\left(s-1+m_{j}-z\right) !}-\sum_{u=1}^{m_{j}-z} \frac{\left(x-x_{j}\right)^{u-1}}{(u-1) !} \frac{x_{j}^{m_{j}-z+s-u}}{\left(m_{j}-z+s-u\right) !}\right) y^{\left(s+m_{j}-1\right)}(0) \\
+ & \frac{\left(x-x_{j}\right)^{m_{j}-z}}{\left(m_{j}-z\right) !} y^{\left(m_{j}\right)}(0)+\sum_{w=1}^{m_{j}-z} \frac{\left(x-x_{j}\right)^{w-1}}{(w-1) !} b_{z+w, j}
\end{aligned}
$$

$y^{\left(m_{j}\right)}(0), y^{\left(m_{j}+1\right)}(0), \ldots, y^{(r-1)}(0)$ in Eq. (3.6) can be obtained the following algebraic equations system

$$
\begin{aligned}
& \sum_{s=2}^{r-m_{j}}\left(\frac{x_{k+2}^{s-1+m_{j}}-x_{k+1}^{s-1+m_{j}}}{\left(s-1+m_{j}\right) !}-\sum_{u=1}^{m_{j}} \frac{\left(x_{k+2}-x_{j}\right)^{u}-\left(x_{k+1}-x_{j}\right)^{u}}{u !} \frac{x_{j}^{m_{j}+s-u}}{\left(m_{j}+s-u\right) !}\right) y^{\left(s+m_{j}-1\right)}(0) \\
& +\left(\frac{\left(x_{k+2}-x_{j}\right)^{m_{j}}-\left(x_{k+1}-x_{j}\right)^{m_{j}}}{m_{j} !}\right) y^{\left(m_{j}\right)}(0)=-b_{r}-\sum_{w=1}^{m_{j}}\left(\frac{\left(x_{k+2}-x_{j}\right)^{w-1}-\left(x_{k+1}-x_{j}\right)^{w-1}}{(w-1) !}\right) b_{w, j} \\
& -C^{T}\left(P_{1} \ldots P_{r} \Psi_{r}\left(x_{k+2}\right)-P_{1} P_{2} \ldots P_{r} \Psi_{r}\left(x_{k+1}\right)-\sum_{h=1}^{m_{j}}\left(\frac{\left(x_{k+2}-x_{j}\right)^{h-1}-\left(x_{k+1}-x_{j}\right)^{h-1}}{(h-1) !} P_{1} \ldots P_{r+1-h} \Psi_{r+1-h}\left(x_{j}\right)\right)\right)
\end{aligned}
$$

where $m_{1}+m_{2}+\ldots+m_{\beta}=r-1$. 
Proof. By successively integrating Eq. (3.5) from $x_{j}$ to $x$ and using boundary conditions

$$
y^{(i-1)}\left(x_{j}\right)=b_{i, j}, 1 \leq i \leq m_{j}, 1 \leq j \leq \beta
$$

the following expressions are obtained:

$$
\begin{aligned}
& y^{\left(m_{j}-1\right)}(x)=C^{T} P_{1} P_{2} P_{3} \ldots P_{r-m_{j}+1}\left(\Psi_{r-m_{j}+1}(x)-\Psi_{r-m_{j}+1}\left(x_{j}\right)\right) \\
& +\left(x-x_{j}\right) y^{\left(m_{j}\right)}(0)+\sum_{s=2}^{r-m_{j}} \frac{x^{s}-x_{j}^{*}}{s !} y^{\left(s+m_{j}-1\right)}(0)+b_{m_{j}, j} \\
& y^{\left(m_{j}-2\right)}(x)=C^{T}\left(P_{1} \ldots P_{r-m_{j}+2}\left(\Psi_{r-m_{j}+2}(x)-\Psi_{r-m_{j}+2}\left(x_{j}\right)\right)-\left(x-x_{j}\right) P_{1} \ldots P_{r-m_{j}+1} \Psi_{r-m_{j}+1}\left(x_{j}\right)\right) \\
& +\frac{\left(x-x_{j}\right)^{2}}{2} y^{\left(m_{j}\right)}(0)+\sum_{s=2}^{r-m_{j}}\left(\frac{x^{s+1}-x_{j}^{s+1}}{(s+1) !}-\frac{\left(x-x_{j}\right) x_{j}^{s}}{s !}\right) y^{\left(s+m_{j}-1\right)}(0)+\left(x-x_{j}\right) b_{m_{j}, j}+b_{m_{j}-1, j} \\
& y^{(z)}(x)=C^{T}\left(P_{1} P_{2} \ldots P_{r-z} \Psi_{r-z}(x)-\sum_{h=1}^{m_{j}-z} \frac{\left(x-x_{j}\right)^{h-1}}{(h-1) !} P_{1} P_{2} \ldots P_{r+1-z-h} \Psi_{r+1-z-h}\left(x_{j}\right)\right) \\
& +\sum_{s=2}^{r-m_{j}}\left(\frac{x^{s-1+m_{j}-z}}{\left(s-1+m_{j}-z\right) !}-\sum_{u=1}^{m_{j}-z} \frac{\left(x-x_{j}\right)^{u-1}}{(u-1) !} \frac{x_{j}^{m_{j}-z+s-u}}{\left(m_{j}-z+s-u\right) !}\right) y^{\left(s+m_{j}-1\right)}(0) \\
& +\frac{\left(x-x_{j}\right)^{m_{j}-z}}{\left(m_{j}-z\right) !} y^{\left(m_{j}\right)}(0)+\sum_{w=1}^{m_{j}-z} \frac{\left(x-x_{j}\right)^{w-1}}{(w-1) !} b_{z+w, j}
\end{aligned}
$$

where $z=0,1, \ldots, m_{j}-1$. This is Eq. (3.6) given in Theorem 3.1. For $z=0$, the following equation can be obtained.

$$
\begin{aligned}
y(x) & =C^{T}\left(P_{1} P_{2} \ldots P_{r} \Psi_{r}(x)-\sum_{h=1}^{m_{j}} \frac{\left(x-x_{j}\right)^{h-1}}{(h-1) !} P_{1} P_{2} \ldots P_{r+1-h} \Psi_{r+1-h}\left(x_{j}\right)\right) \\
& +\sum_{s=2}^{r-m_{j}}\left(\frac{x^{s-1+m_{j}}}{\left(s-1+m_{j}\right) !}-\sum_{u=1}^{m_{j}} \frac{\left(x-x_{j}\right)^{u}}{u !} \frac{x_{j}^{m_{j}+s-u}}{\left(m_{j}+s-u\right) !}\right) y^{\left(s+m_{j}-1\right)}(0) \\
& +\frac{\left(x-x_{j}\right)^{m_{j}}}{m_{j} !} y^{\left(m_{j}\right)}(0)+\sum_{w=1}^{m_{j}} \frac{\left(x-x_{j}\right)^{w-1}}{(w-1) !} b_{w, j}
\end{aligned}
$$

If boundary condition $y\left(x_{\beta+1}\right)-y\left(x_{\beta+2}\right)=b_{r}$ can be satisfied, the following equation can be obtained as:

$$
\begin{aligned}
& \sum_{s=2}^{r-m_{j}}\left(\frac{x_{k+2}^{s-1+m_{j}}-x_{k+1}^{s-1+m_{j}}}{\left(s-1+m_{j}\right) !}-\sum_{u=1}^{m_{j}} \frac{\left(x_{k+2}-x_{j}\right)^{u}-\left(x_{k+1}-x_{j}\right)^{u}}{u !} \frac{x_{j}^{m_{j}+s-u}}{\left(m_{j}+s-u\right) !}\right) y^{\left(s+m_{j}-1\right)}(0) \\
& +\left(\frac{\left(x_{k+2}-x_{j}\right)^{m_{j}}-\left(x_{k+1}-x_{j}\right)^{m_{j}}}{m_{j} !}\right) y^{\left(m_{j}\right)}(0)=-b_{r}-\sum_{w=1}^{m_{j}}\left(\frac{\left(x_{k+2}-x_{j}\right)^{w-1}-\left(x_{k+1}-x_{j}\right)^{w-1}}{(w-1) !}\right) b_{w, j} \\
& -C^{T}\left(P_{1} \ldots P_{r} \Psi_{r}\left(x_{k+2}\right)-P_{1} P_{2} \ldots P_{r} \Psi_{r}\left(x_{k+1}\right)-\sum_{h=1}^{m_{j}}\left(\frac{\left(x_{k+2}-x_{j}\right)^{h-1}-\left(x_{k+1}-x_{j}\right)^{h-1}}{(h-1) !} P_{1} \ldots P_{r+1-h} \Psi_{r+1-h}\left(x_{j}\right)\right)\right)
\end{aligned}
$$

where $m_{1}+m_{2}+\ldots+m_{\beta}=r-1$.

Conclusion 3.2. If $\beta=1$ then $j=1$ and $m_{1}=r-1$ are obtained. Hence

$$
\begin{gathered}
y(x)=C^{T}\left(P_{1} P_{2} \ldots P_{r} \Psi_{r}(x)-\sum_{h=1}^{r-1} \frac{\left(x-x_{1}\right)^{h-1}}{(h-1) !} P_{1} P_{2} \ldots P_{r+1-h} \Psi_{r+1-h}\left(x_{1}\right)\right) \\
+\frac{\left(x-x_{1}\right)^{r-1}}{(r-1) !} y^{(r-1)}(0)+\sum_{w=1}^{r-1} \frac{\left(x-x_{1}\right)^{w-1}}{(w-1) !} b_{w, 1}
\end{gathered}
$$

is obtained, where $y^{(t-1)}(0)$ is obtain as

$$
\begin{aligned}
& \left(\frac{\left(x_{3}-x_{1}\right)^{r-1}-\left(x_{2}-x_{1}\right)^{r-1}}{(r-1) !}\right) y^{(r-1)}(0)=-b_{r}-\sum_{w=1}^{r-1}\left(\frac{\left(x_{3}-x_{1}\right)^{w-1}-\left(x_{2}-x_{2}\right)^{w-1}}{(w-1) !}\right) b_{w, 1} \\
& -C^{T}\left(P_{1} \ldots P_{r} \Psi_{r}\left(x_{3}\right)-P_{1} P_{2} \ldots P_{r} \Psi_{r}\left(x_{2}\right)-\sum_{h=1}^{r-1}\left(\frac{\left(x_{3}-x_{1}\right)^{h-1}-\left(x_{2}-x_{j}\right)^{h-1}}{(h-1) !} P_{1} \ldots P_{r+1-h} \Psi_{r+1-h}\left(x_{1}\right)\right)\right)
\end{aligned}
$$

Replacing (3.1)-(3.6) into Eq. (1.1) or Eq. (1.2), we have linear or nonlinear algebraic equations respectively. The collocation points can be taken as $2^{k+1} x_{n i}-2 n+1=\cos \frac{((M+1)-i) \pi}{(M+1)}$ or

$$
x_{n i}=\frac{1}{2^{k+1}}\left(2 n-1+\cos \frac{((M+1)-i) \pi}{(M+1)}\right), i=1,2, \ldots, M, n=1,2, \ldots, 2^{k}
$$

which are also called the turning points of $T_{M+1}\left(2^{k+1} x-2 n+1\right)$. Substituting the Chebyshev collocation points into linear or nonlinear algebraic equations, a discretizised form of the vectors $\Psi\left(x_{n i}\right), \Psi_{1}\left(x_{n i}\right)$ and $\Psi_{r}\left(x_{n i}\right)$ can be obtained. Hence, we obtain linear or nonlinear algebraic equations systems. By solving linear or nonlinear algebraic equation systems, we can find the coefficients of the Chebyshev wavelet series that satisfied differential equation and its initial or boundary conditions. 


\section{Error analysis}

For error analysis of Chebyshev wavelet method, the following Lemma and Theorems are given.

Lemma 4.1. (See [36]) If the Chebyshev wavelet expansion of a continuous function $f(x)$ converges uniformly, then the Chebyshev wavelet expansion converges to a function $f(x)$.

Theorem 4.2. (See [36]) A function $f(x) \in L_{\omega}^{2}([0,1])$ with bounded second derivative $\left|f^{\prime \prime}(x)\right| \leq N$, can be expanded as an infinite sum of Chebyshev wavelets, and the series converges uniformly to $f(x)$. That is

$$
f(x)=\sum_{n=1}^{\infty} \sum_{m=0}^{\infty} f_{n m} \psi_{n m}(x) .
$$

Since the truncated Chebyshev wavelets series

$$
C^{T} \Psi(x)=\sum_{n=1}^{2^{k}} \sum_{m=0}^{M-1} c_{n m} \psi_{n m}(x)
$$

is an approximate solution of given problem and $y(x)$ is an exact solution, an error function $f(x)$ can be given as:

$$
E(x)=\left|y(x)-C^{T} \Psi(x)\right|
$$

The error bound of the approximate solution obtained by using truncated Chebyshev wavelets series is given by the following theorem.

Theorem 4.3. (See [37]) Suppose that $y(x) \in C^{m}[0,1]$ and $C^{T} \Psi(x)$ is the approximate solution of problem using the Chebyshev wavelets method. Then the error bound can be obtained as follows:

$$
E(x) \leq\left\|\frac{2}{m ! 4^{m} 2^{m(k-1)}} \max _{x \in[0,1]}\left|y^{(m)}(x)\right|\right\|^{2} .
$$

\section{Numerical results}

Example 5.1. Consider the forth order linear boundary value problem [19]

$$
\left\{\begin{array}{l}
y^{(4)}(x)-e^{x} y^{\prime \prime \prime}(x)+y(x)=1-e^{x} \cosh (x)+2 \sinh (x), \quad 0 \leq x \leq 1 \\
y\left(\frac{1}{4}\right)=1+\sinh \left(\frac{1}{4}\right), \quad y^{\prime}\left(\frac{1}{4}\right)=\cosh \left(\frac{1}{4}\right), \\
y^{\prime \prime}\left(\frac{1}{4}\right)=\sinh \left(\frac{1}{4}\right), \quad y\left(\frac{1}{2}\right)-y\left(\frac{3}{4}\right)=\sinh \left(\frac{1}{2}\right)-\sinh \left(\frac{3}{4}\right) .
\end{array}\right.
$$

with analytic solution $y(x)=1+\sinh (x)$. It is assumed that $y^{4}(x)$ can be expanded in terms of truncated Chebyshev wavelet series as

$$
y^{(4)}(x)=\sum_{n=1}^{2^{k}} \sum_{m=0}^{M-1} f_{n m} \psi_{n m}(x)=C^{T} \Psi(x)
$$

By integrating this equation from 0 to xand using boundary condition,

$$
y^{\prime \prime \prime}(x)=\int_{0}^{x} C^{T} \Psi(s) d s+y^{\prime \prime \prime}(0)=C^{T} P_{1} \Psi_{1}(x)+y^{\prime \prime \prime}(0)
$$

is obtained. By integrating this equation three times from $\frac{1}{4}$ to $x$ and using boundary conditions, following equations are obtained.

$$
\begin{gathered}
y^{\prime \prime}(x)=C^{T}\left(P_{1} P_{2} \Psi_{2}(x)-P_{1} P_{2} \Psi_{2}\left(\frac{1}{4}\right)\right)+\left(x-\frac{1}{4}\right) y^{\prime \prime \prime}(0)+\sinh \left(\frac{1}{4}\right) \\
y^{\prime}(x)=C^{T} P_{1} P_{2}\left(P_{3} \Psi_{3}(x)-P_{3} \Psi_{3}\left(\frac{1}{4}\right)-\left(x-\frac{1}{4}\right) \Psi_{2}\left(\frac{1}{4}\right)\right)+\frac{\left(x-\frac{1}{4}\right)^{2}}{2} y^{\prime \prime \prime}(0)+\left(x-\frac{1}{4}\right) \sinh \left(\frac{1}{4}\right)+\cosh \left(\frac{1}{4}\right) \\
y(x)=C^{T}\left(P_{1} P_{2} P_{3} P_{4} \Psi_{4}(x)-P_{1} P_{2} P_{3} P_{4} \Psi_{4}\left(\frac{1}{4}\right)-\left(x-\frac{1}{4}\right) P_{1} P_{2} P_{3} \Psi_{3}\left(\frac{1}{4}\right)-\frac{\left(x-\frac{1}{4}\right)^{2}}{2} P_{1} P_{2} \Psi_{2}\left(\frac{1}{4}\right)\right) \\
+\frac{\left(x-\frac{1}{4}\right)^{3}}{3 !} y^{\prime \prime \prime}(0)+\frac{\left(x-\frac{1}{4}\right)^{2}}{2 !} \sinh \left(\frac{1}{4}\right)+\left(x-\frac{1}{4}\right) \cosh \left(\frac{1}{4}\right)+1+\sinh \left(\frac{1}{4}\right)
\end{gathered}
$$

By using boundary condition $y\left(\frac{1}{2}\right)-y\left(\frac{3}{4}\right)=\sinh \left(\frac{1}{2}\right)-\sinh \left(\frac{3}{4}\right), y^{\prime \prime \prime}(0)$ is obtained as:

$$
\begin{gathered}
y^{\prime \prime \prime}(0)=\frac{384}{7} C^{T}\left(P_{1} P_{2} P_{3} P_{4} \Psi_{4}\left(\frac{1}{2}\right)-P_{1} P_{2} P_{3} P_{4} \Psi_{4}\left(\frac{3}{4}\right)+\frac{1}{4} P_{1} P_{2} P_{3} \Psi_{3}\left(\frac{1}{4}\right)+\frac{3}{32} P_{1} P_{2} \Psi_{2}\left(\frac{1}{4}\right)\right) \\
-\frac{384}{7}\left(\sinh \left(\frac{1}{2}\right)-\sinh \left(\frac{3}{4}\right)+\frac{1}{4} \cosh \left(\frac{1}{4}\right)+\frac{3}{32} \sinh \left(\frac{1}{4}\right)\right)
\end{gathered}
$$

Hence, replacing Eq. (5.5) into the Eqs. (5.3) and (5.4), we have 
Table 1: Comparisons of the absolute errors of [19] and proposed method for various values of $M, k$ and $x$

\begin{tabular}{|l|l|l|l|l|l|l|l|}
\hline$x$ & $M=4, k=0$ & $M=4, k=1$ & $M=4, k=2$ & $M=8, k=0$ & $M=16, k=0$ & $\left|y-y_{101}\right|[19]$ & $\left|y-y_{151}\right|[19]$ \\
\hline 0 & $1.57537 \mathrm{e}-8$ & $4.63339 \mathrm{e}-10$ & $2.61472 \mathrm{e}-11$ & $6.32711 \mathrm{e}-15$ & $1 \mathrm{e}-19$ & 0.0000255 & $1.13356 \mathrm{E}-6$ \\
\hline 0.1 & $2.38097 \mathrm{e}-9$ & $1.04070 \mathrm{e}-10$ & $5.93744 \mathrm{e}-12$ & $3.38417 \mathrm{e}-15$ & $1 \mathrm{e}-19$ & $4.53581 \mathrm{E}-6$ & $2.01715 \mathrm{E}-7$ \\
\hline 0.2 & $3.88574 \mathrm{e}-11$ & $3.13685 \mathrm{e}-12$ & $2.31892 \mathrm{e}-13$ & $2.12340 \mathrm{e}-16$ & $3 \mathrm{e}-19$ & $1.32679 \mathrm{E}-7$ & $5.90784 \mathrm{E}-9$ \\
\hline 0.3 & $5.15129 \mathrm{e}-12$ & $1.90014 \mathrm{e}-12$ & $1.48048 \mathrm{e}-14$ & $2.20110 \mathrm{e}-17$ & $2 \mathrm{e}-19$ & $9.91385 \mathrm{E}-8$ & $4.39712 \mathrm{E}-9$ \\
\hline 0.4 & $9.21096 \mathrm{e}-10$ & $2.86581 \mathrm{e}-11$ & $8.51610 \mathrm{e}-13$ & $4.11361 \mathrm{e}-15$ & $3 \mathrm{e}-19$ & $1.90635 \mathrm{E}-6$ & $8.46552 \mathrm{E}-8$ \\
\hline 0.5 & $5.55451 \mathrm{e}-9$ & $1.46005 \mathrm{e}-10$ & $5.70554 \mathrm{e}-12$ & $8.99446 \mathrm{e}-15$ & $1 \mathrm{e}-19$ & $5.92446 \mathrm{E}-6$ & $2.63147 \mathrm{E}-7$ \\
\hline 0.6 & $1.28454 \mathrm{e}-8$ & $4.31240 \mathrm{e}-10$ & $1.38779 \mathrm{e}-11$ & $8.47493 \mathrm{e}-15$ & $2 \mathrm{e}-19$ & $9.75828 \mathrm{E}-6$ & $4.33469 \mathrm{E}-7$ \\
\hline 0.7 & $1.30931 \mathrm{e}-8$ & $3.93193 \mathrm{e}-10$ & $1.42221 \mathrm{e}-11$ & $7.79239 \mathrm{e}-15$ & $2 \mathrm{e}-19$ & $9.32982 \mathrm{E}-6$ & $4.14438 \mathrm{E}-7$ \\
\hline 0.8 & $9.97851 \mathrm{e}-9$ & $3.11259 \mathrm{e}-10$ & $1.24207 \mathrm{e}-11$ & $1.01678 \mathrm{e}-14$ & $2 \mathrm{e}-19$ & $5.99989 \mathrm{E}-7$ & $2.67207 \mathrm{E}-8$ \\
\hline 0.9 & $7.17676 \mathrm{e}-8$ & $2.30928 \mathrm{e}-9$ & $1.02985 \mathrm{e}-10$ & $8.93500 \mathrm{e}-15$ & 0 & 0.0000265 & $1.17736 \mathrm{E}-6$ \\
\hline 1.0 & $1.81832 \mathrm{e}-7$ & $6.63219 \mathrm{e}-9$ & $3.16336 \mathrm{e}-10$ & $6.03590 \mathrm{e}-15$ & 0 & 0.0000765 & $3.39732 \mathrm{E}-6$ \\
\hline
\end{tabular}

$$
\begin{gathered}
y^{\prime \prime \prime}(x)=C^{T}\left(P_{1} \Psi_{1}(x)+\frac{384}{7} C^{T} P_{1} P_{2}\left(P_{3} P_{4} \Psi_{4}\left(\frac{1}{2}\right)-P_{3} P_{4} \Psi_{4}\left(\frac{3}{4}\right)+\frac{1}{4} P_{3} \Psi_{3}\left(\frac{1}{4}\right)+\frac{3}{32} \Psi_{2}\left(\frac{1}{4}\right)\right)\right) \\
-\frac{384}{7}\left(\sinh \left(\frac{1}{2}\right)-\sinh \left(\frac{3}{4}\right)+\frac{1}{4} \cosh \left(\frac{1}{4}\right)+\frac{3}{32} \sinh \left(\frac{1}{4}\right)\right) \\
y(x)=C^{T}\left(\begin{array}{c}
P_{1} P_{2} P_{3} P_{4} \Psi_{4}(x)-P_{1} P_{2} P_{3} P_{4} \Psi_{4}\left(\frac{1}{4}\right)-\left(x-\frac{1}{4}\right) P_{1} P_{2} P_{3} \Psi_{3}\left(\frac{1}{4}\right)-\frac{\left(x-\frac{1}{4}\right)^{2}}{2} P_{1} P_{2} \Psi_{2}\left(\frac{1}{4}\right) \\
+\frac{384\left(x-\frac{1}{4}\right)^{3}}{42}\left(P_{1} P_{2} P_{3} P_{4} \Psi_{4}\left(\frac{1}{2}\right)-P_{1} P_{2} P_{3} P_{4} \Psi_{4}\left(\frac{3}{4}\right)+\frac{1}{4} P_{1} P_{2} P_{3} \Psi_{3}\left(\frac{1}{4}\right)+\frac{3}{32} P_{1} P_{2} \Psi_{2}\left(\frac{1}{4}\right)\right)
\end{array}\right) \\
-\quad-\frac{384\left(x-\frac{1}{4}\right)^{3}}{42}\left(\sinh \left(\frac{1}{2}\right)-\sinh \left(\frac{3}{4}\right)+\frac{1}{4} \cosh \left(\frac{1}{4}\right)+\frac{3}{32} \sinh \left(\frac{1}{4}\right)\right)+\frac{\left(x-\frac{1}{4}\right)^{2}}{2} \sinh \left(\frac{1}{4}\right) \\
+\left(x-\frac{1}{4}\right) \cosh \left(\frac{1}{4}\right)+1+\sinh \left(\frac{1}{4}\right)
\end{gathered}
$$

Replacing Eqs. (5.2), (5.6) and (5.7) into Eq. (5.1), we have

$$
\begin{gathered}
C^{T}\left(\begin{array}{c}
\Psi(x)+P_{1} P_{2} P_{3} P_{4}\left(\Psi_{4}(x)-\Psi_{4}\left(\frac{1}{4}\right)\right)-\left(x-\frac{1}{4}\right) P_{1} P_{2} P_{3} \Psi_{3}\left(\frac{1}{4}\right)-\frac{\left(x-\frac{1}{4}\right)^{2}}{2} P_{1} P_{2} \Psi_{2}\left(\frac{1}{4}\right)-e^{x} P_{1} \Psi_{1}(x) \\
+\frac{384}{7}\left(\frac{\left(x-\frac{1}{4}\right)^{3}}{6}-e^{x}\right)\left(P_{1} P_{2} P_{3} P_{4} \Psi_{4}\left(\frac{1}{2}\right)-P_{1} P_{2} P_{3} P_{4} \Psi_{4}\left(\frac{3}{4}\right)+\frac{1}{4} P_{1} P_{2} P_{3} \Psi_{3}\left(\frac{1}{4}\right)+\frac{3}{32} P_{1} P_{2} \Psi_{2}\left(\frac{1}{4}\right)\right)
\end{array}\right) \\
=\frac{384}{7}\left(\frac{\left(x-\frac{1}{4}\right)^{3}}{6}-e^{x}\right)\left(\sinh \left(\frac{1}{2}\right)-\sinh \left(\frac{3}{4}\right)+\frac{1}{4} \cosh \left(\frac{1}{4}\right)+\frac{3}{32} \sinh \left(\frac{1}{4}\right)\right)-\frac{\left(x-\frac{1}{4}\right)^{2}}{2} \sinh \left(\frac{1}{4}\right) \\
-\left(x-\frac{1}{4}\right) \cosh \left(\frac{1}{4}\right)-\sinh \left(\frac{1}{4}\right)-e^{x} \cosh (x)+2 \sinh (x)
\end{gathered}
$$

Algebraic equation system achieved in Eq. (5.8) by using Chebyshev collocation points can be solved and the coefficients $C^{T}$ in Eq. (5.7) which is satisfied differential equation and whose boundary conditions can be obtained. Table 1 shows the absolute errors for $M=4, k=0$, $M=4, k=1, M=4, k=2, M=8, k=0$ and $M=16, k=0$. As can be seen in Table 1, the results obtained by the proposed method are superior from Reproducing Kernel Method [19] for small grid points such as $M=4, k=0$.

Example 5.2. Consider the second order nonlinear boundary value problem [21].

$$
\left\{\begin{array}{l}
y^{\prime \prime}(x)+\frac{\sqrt{2}}{8} y(x)+\frac{1}{32} y^{2}(x)=\frac{32}{9} x^{2}-\frac{16}{3} x, \quad 0 \leq x \leq 1 \\
y(0)=0, \quad y(1)-y\left(\frac{1}{2}\right)=0
\end{array}\right.
$$

It is assumed that $y^{\prime \prime}(x)$ can be expanded in terms of truncated Chebyshev wavelet series as

$$
y^{\prime \prime}(x)=\sum_{n=1}^{2^{k}} \sum_{m=0}^{M-1} f_{n m} \psi_{n m}(x)=C^{T} \Psi(x)
$$

Similar process given in Example 5.1, the following equations can be obtained

$$
\begin{gathered}
y(x)=C^{T}\left(P_{1} P_{2} \Psi_{2}(x)-2 x P_{1} P_{2} \Psi_{2}(1)+2 x P_{1} P_{2} \Psi_{2}\left(\frac{1}{2}\right)\right) \\
C^{T}\left(\Psi(x)+\frac{\sqrt{2}}{8} P_{1} P_{2} \Psi_{2}(x)-\frac{x \sqrt{2}}{4} P_{1} P_{2} \Psi_{2}(1)+\frac{x \sqrt{2}}{4} P_{1} P_{2} \Psi_{2}\left(\frac{1}{2}\right)\right) \\
+\frac{1}{32}\left(C^{T}\left(P_{1} P_{2} \Psi_{2}(x)-2 x P_{1} P_{2} \Psi_{2}(1)+2 x\left(x-\frac{1}{4}\right) P_{1} P_{2} \Psi_{2}\left(\frac{1}{2}\right)\right)\right)^{2}-\frac{32}{9} x^{2}+\frac{16}{3} x=0
\end{gathered}
$$

Nonlinear algebraic equation system achieved from Eq. (5.11) by using collocation points can be solved and the coefficients $C^{T}$ in Eq. (5.10) which is satisfied differential equation and whose boundary conditions are obtained. Table 2 shows the approximate solutions for $M=4, k=0, M=4, k=1, M=4, k=2, M=8, k=0$ and $M=16, k=0$. As can be seen in Table 2 that the results obtained by the proposed method are satisfied the boundary condition $y(1)-y\left(\frac{1}{2}\right)=0$. When number of grid points increase, the precisions of approximate solutions increase. The results obtained by the proposed method for small grid points such as $M=4, k=0$ are also superior from Monotone Iterative Method [21] not satisfying the boundary condition $y(1)-y\left(\frac{1}{2}\right)=0$ exactly 
Table 2: Approximate solutions of proposed method for various values of $M, k$ and $x$ and [21]

\begin{tabular}{|l|l|l|l|l|l|l|}
\hline$x$ & $M=4, k=0$ & $M=4, k=1$ & $M=4, k=2$ & $M=8, k=0$ & $M=16, k=0$ & {$[21]$ Third Term } \\
\hline 0 & 0.260929 & 0.949067 & 0.157524 & 0.260974 & 0.260974 e-19 & 0 \\
& $\mathrm{e}-19$ & $\mathrm{e}-21$ & $\mathrm{e}-21$ & 0.19 & & \\
\hline 0.1 & 0.1035508429 & 0.1035501266 & 0.1035502791 & 0.1035502920 & 0.1035502920 & 0.1040764497 \\
\hline 0.2 & 0.2019974623 & 0.2019955359 & 0.2019958847 & 0.2019959102 & 0.2019959103 & 0.2027652843 \\
\hline 0.3 & 0.2908872022 & 0.2908870108 & 0.2908875266 & 0.2908875633 & 0.2908875632 & 0.2916751511 \\
\hline 0.4 & 0.3664960100 & 0.3664989187 & 0.3664995714 & 0.3664996188 & 0.3664996189 & 0.3671584606 \\
\hline 0.5 & 0.4258337040 & 0.4258381953 & 0.4258390441 & 0.4258391011 & 0.4258391014 & 0.4263068077 \\
\hline 0.6 & 0.4666492402 & 0.4666527782 & 0.4666535640 & 0.4666536253 & 0.4666536254 & 0.4669454120 \\
\hline 0.7 & 0.4874359792 & 0.4874368797 & 0.4874377503 & 0.4874378143 & 0.4874378143 & 0.4876278426 \\
\hline 0.8 & 0.4874369540 & 0.4874368797 & 0.4874377503 & 0.4874378140 & 0.4874378143 & 0.4876319741 \\
\hline 0.9 & 0.4666501363 & 0.4666527782 & 0.4666535639 & 0.4666536252 & 0.4666536254 & 0.4669578065 \\
\hline 1.0 & 0.4258337040 & 0.4258381953 & 0.4258390441 & 0.4258391011 & 0.4258391014 & 0.4263274652 \\
\hline
\end{tabular}

Table 3: Approximate solutions of proposed method for various values of $M, k$ and $x$ and [17]

\begin{tabular}{|c|l|l|l|l|l|l|}
\hline$x$ & $M=4, k=0$ & $M=4, k=2$ & $M=16, k=0$ & $M=16, k=1$ & $\begin{array}{l}{[17] \text { OHAM }} \\
\text { Second Order }\end{array}$ & $\begin{array}{l}\text { [17] HPM } \\
\text { Second Order }\end{array}$ \\
\hline 0 & $\begin{array}{l}0.188293 \\
\mathrm{e}-19\end{array}$ & $\begin{array}{l}0.734306 \\
\mathrm{e}-23\end{array}$ & $0.188296 \mathrm{e}-19$ & $0.129858 \mathrm{e}-20$ & 0 & 0 \\
\hline 0.1 & 0.0656100870 & 0.0656099762 & 0.0656099772 & 0.0656099772 & 0.0656099707 & 0.0655919115 \\
\hline 0.2 & 0.1209706322 & 0.1209703634 & 0.1209703654 & 0.1209703653 & 0.1209703640 & 0.1209353047 \\
\hline 0.3 & 0.1658758980 & 0.1658757275 & 0.1658757303 & 0.1658757303 & 0.1658757339 & 0.1658256598 \\
\hline 0.4 & 0.2001594201 & 0.2001594622 & 0.2001594656 & 0.2001594656 & 0.2001594697 & 0.2000971743 \\
\hline 0.5 & 0.2236942481 & 0.2236943874 & 0.2236943913 & 0.2236943913 & 0.2236943923 & 0.2236233202 \\
\hline 0.6 & 0.2363931859 & 0.2363932068 & 0.2363932109 & 0.2363932109 & 0.2363932086 & 0.2363172683 \\
\hline 0.7 & 0.2382090332 & 0.2382088205 & 0.2382088245 & 0.2382088245 & 0.2382088217 & 0.2381321777 \\
\hline 0.8 & 0.2291348258 & 0.2291344944 & 0.2291344982 & 0.2291344982 & 0.2291344982 & 0.2290613518 \\
\hline 0.9 & 0.2092040768 & 0.2092038847 & 0.2092038882 & 0.2092038882 & 0.2092038920 & 0.2091382608 \\
\hline 1.0 & 0.1784910170 & 0.1784909168 & 0.1784909199 & 0.1784909199 & 0.1784909250 & 0.1784364302 \\
\hline
\end{tabular}

Example 5.3. Consider the second order nonlinear boundary value problem [17]

$$
\left\{\begin{array}{l}
y^{\prime \prime}(x)+\frac{3}{8} y(x)+\frac{2}{1089}\left(y^{\prime}(x)\right)^{2}+1=0, \quad 0 \leq x \leq 1 \\
y(0)=0, \quad y(1)-y\left(\frac{1}{3}\right)=0 .
\end{array}\right.
$$

It is assumed that $y^{\prime \prime}(x)$ can be expanded in terms of truncated Chebyshev wavelet series as

$$
y^{\prime \prime}(x)=\sum_{n=1}^{2^{k}} \sum_{m=0}^{M-1} f_{n m} \psi_{n m}(x)=C^{T} \Psi(x)
$$

Similar process given in Example 5.1, the following equations can be obtained

$$
\begin{gathered}
y(x)=C^{T}\left(P_{1} P_{2} \Psi_{2}(x)-\frac{3 x}{2} P_{1} P_{2} \Psi_{2}(1)+\frac{3 x}{2} P_{1} P_{2} \Psi_{2}\left(\frac{1}{3}\right)\right) \\
C^{T}\left(\Psi(x)+\frac{3}{8} P_{1} P_{2} \Psi_{2}(x)-\frac{9 x}{16} P_{1} P_{2} \Psi_{2}(1)+\frac{9 x}{16} P_{1} P_{2} \Psi_{2}\left(\frac{1}{3}\right)\right) \\
+\frac{2}{1089}\left(C^{T}\left(P_{1} \Psi_{1}(x)-\frac{3}{2} P_{1} P_{2} \Psi_{2}(1)+\frac{3}{2} P_{1} P_{2} \Psi_{2}\left(\frac{1}{3}\right)\right)\right)^{2}+1=0
\end{gathered}
$$

Nonlinear algebraic equation system achieved from Eq. (5.14) by using collocation points can be solved and the coefficients $C^{T}$ in Eq. (5.13) satisfied differential equation and whose boundary conditions are obtained. Table 3 shows the approximate solutions for $M=4, k=0$, $M=4, k=2, M=16, k=0$ and $M=16, k=1$. As can be seen in Table 3, the precisions of approximate solutions obtained by the proposed method increase when number of grid points increase. The results obtained by the proposed method for small grid points such as $M=4, k=0$ are superior from Homotopy Perturbation Method and Optimal Homotopy Asymptotic Method in [17].

Example 5.4. Consider the forth order nonlinear boundary value problem

$$
\left\{\begin{array}{l}
y^{(4)}(x)-\sin (x) y^{\prime \prime}(x)+y(x)+\sin (y(x))=1+\sin (1+\sin (x))+(2+\sin (x)) \sin (x), 0 \leq x \leq 1 \\
y\left(\frac{1}{4}\right)=1+\sin \left(\frac{1}{4}\right), \quad y^{\prime}\left(\frac{1}{4}\right)=\cos \left(\frac{1}{4}\right), \\
y^{\prime \prime}\left(\frac{1}{4}\right)=-\sin \left(\frac{1}{4}\right), \quad y\left(\frac{1}{2}\right)-y\left(\frac{3}{4}\right)=\sin \left(\frac{1}{2}\right)-\sin \left(\frac{3}{4}\right) .
\end{array}\right.
$$

with analytic solution $y(x)=1+\sin (x)$. 
Table 4: The absolute errors of proposed method for various values of $M, k$ and $x$

\begin{tabular}{|l|l|l|l|l|l|}
\hline$x$ & $M=4, k=0$ & $M=4, k=1$ & $M=4, k=2$ & $M=8, k=0$ & $M=16, k=0$ \\
\hline 0 & $1.305827 \mathrm{e}-8$ & $4.55634 \mathrm{e}-10$ & $2.69793 \mathrm{e}-11$ & $6.48270 \mathrm{e}-15$ & $1.0 \mathrm{e}-19$ \\
\hline 0.1 & $1.889004 \mathrm{e}-9$ & $9.89746 \mathrm{e}-11$ & $5.96046 \mathrm{e}-12$ & $3.14499 \mathrm{e}-15$ & $7.0 \mathrm{e}-21$ \\
\hline 0.2 & $2.754407 \mathrm{e}-11$ & $2.85912 \mathrm{e}-12$ & $2.25583 \mathrm{e}-13$ & $1.87940 \mathrm{e}-16$ & $6.0 \mathrm{e}-20$ \\
\hline 0.3 & $8.825138 \mathrm{e}-12$ & $1.59099 \mathrm{e}-12$ & $1.50140 \mathrm{e}-14$ & $1.88610 \mathrm{e}-16$ & $1.0 \mathrm{e}-20$ \\
\hline 0.4 & $8.503094 \mathrm{e}-10$ & $2.02999 \mathrm{e}-11$ & $8.22951 \mathrm{e}-13$ & $3.39057 \mathrm{e}-15$ & $3.0 \mathrm{e}-20$ \\
\hline 0.5 & $4.775323 \mathrm{e}-9$ & $1.04675 \mathrm{e}-10$ & $5.14514 \mathrm{e}-12$ & $6.96137 \mathrm{e}-15$ & $3.0 \mathrm{e}-20$ \\
\hline 0.6 & $1.061068 \mathrm{e}-8$ & $3.18029 \mathrm{e}-10$ & $1.20212 \mathrm{e}-11$ & $5.91620 \mathrm{e}-15$ & 0 \\
\hline 0.7 & $1.058432 \mathrm{e}-8$ & $2.81752 \mathrm{e}-10$ & $1.20549 \mathrm{e}-11$ & $5.57247 \mathrm{e}-15$ & $1.3 \mathrm{e}-19$ \\
\hline 0.8 & $6.632389 \mathrm{e}-9$ & $2.09432 \mathrm{e}-10$ & $8.86424 \mathrm{e}-12$ & $8.34323 \mathrm{e}-15$ & $7.0 \mathrm{e}-20$ \\
\hline 0.9 & $4.857164 \mathrm{e}-8$ & $1.52893 \mathrm{e}-9$ & $7.46368 \mathrm{e}-11$ & $8.29286 \mathrm{e}-15$ & $1.4 \mathrm{e}-19$ \\
\hline 1.0 & $1.148666 \mathrm{e}-7$ & $4.21506 \mathrm{e}-9$ & $2.18685 \mathrm{e}-10$ & $8.17725 \mathrm{e}-15$ & $1.5 \mathrm{e}-19$ \\
\hline
\end{tabular}

Similar process given in Example 5.1, the following equations can be obtained

$$
\begin{gathered}
y(x)=C^{T}\left(\begin{array}{c}
P_{1} P_{2} P_{3} P_{4} \Psi_{4}(x)-P_{1} P_{2} P_{3} P_{4} \Psi_{4}\left(\frac{1}{4}\right)-\left(x-\frac{1}{4}\right) P_{1} P_{2} P_{3} \Psi_{3}\left(\frac{1}{4}\right)-\frac{\left(x-\frac{1}{4}\right)^{2}}{2} P_{1} P_{2} \Psi_{2}\left(\frac{1}{4}\right) \\
+\frac{384\left(x-\frac{1}{4}\right)^{3}}{42}\left(P_{1} P_{2} P_{3} P_{4} \Psi_{4}\left(\frac{1}{2}\right)-P_{1} P_{2} P_{3} P_{4} \Psi_{4}\left(\frac{3}{4}\right)+\frac{1}{4} P_{1} P_{2} P_{3} \Psi_{3}\left(\frac{1}{4}\right)+\frac{3}{32} P_{1} P_{2} \Psi_{2}\left(\frac{1}{4}\right)\right)
\end{array}\right) \\
-\frac{384\left(x-\frac{1}{4}\right)^{3}}{42}\left(\sin \left(\frac{1}{2}\right)-\sin \left(\frac{3}{4}\right)+\frac{1}{4} \cos \left(\frac{1}{4}\right)-\frac{3}{32} \sin \left(\frac{1}{4}\right)\right)-\frac{\left(x-\frac{1}{4}\right)^{2}}{2} \sin \left(\frac{1}{4}\right)+\left(x-\frac{1}{4}\right) \cos \left(\frac{1}{4}\right)+1+\sin \left(\frac{1}{4}\right) \\
C^{T}\left(\begin{array}{l}
\frac{384}{7}\left(\frac{\left(x-\frac{1}{4}\right)^{3}}{6}-\left(x-\frac{1}{4}\right) \sin (x)\right)\left(P_{1} P_{2} P_{3} P_{4} \Psi_{4}\left(\frac{1}{2}\right)-P_{1} P_{2} P_{3} P_{4} \Psi_{4}\left(\frac{3}{4}\right)+\frac{1}{4} P_{1} P_{2} P_{3} \Psi_{3}\left(\frac{1}{4}\right)+\frac{3}{32} P_{1} P_{2} \Psi_{2}\left(\frac{1}{4}\right)\right) \\
+\Psi(x)+P_{1} P_{2} P_{3} P_{4}\left(\Psi_{4}(x)-\Psi_{4}\left(\frac{1}{4}\right)\right)-\left(x-\frac{1}{4}\right) P_{1} P_{2} P_{3} \Psi_{3}\left(\frac{1}{4}\right)-\frac{\left(x-\frac{1}{4}\right)^{2}}{2} P_{1} P_{2} \Psi_{2}\left(\frac{1}{4}\right) \\
-\sin (x) P_{1} P_{2}\left(\Psi_{2}(x)-\Psi_{2}\left(\frac{1}{4}\right)\right) \\
+\sin \left(\begin{array}{l}
C^{T}\left(\begin{array}{c}
P_{1} P_{2} P_{3} P_{4} \Psi_{4}(x)-P_{1} P_{2} P_{3} P_{4} \Psi_{4}\left(\frac{1}{4}\right)-\left(x-\frac{1}{4}\right) P_{1} P_{2} P_{3} \Psi_{3}\left(\frac{1}{4}\right)-\frac{\left(x-\frac{1}{4}\right)^{2}}{2} P_{1} P_{2} \Psi_{2}\left(\frac{1}{4}\right) \\
+\frac{384\left(x-\frac{1}{4}\right)^{3}}{42}\left(P_{1} P_{2} P_{3} P_{4} \Psi_{4}\left(\frac{1}{2}\right)-P_{1} P_{2} P_{3} P_{4} \Psi_{4}\left(\frac{3}{4}\right)+\frac{1}{4} P_{1} P_{2} P_{3} \Psi_{3}\left(\frac{1}{4}\right)+\frac{3}{32} P_{1} P_{2} \Psi_{2}\left(\frac{1}{4}\right)\right)
\end{array}\right) \\
-\frac{384\left(x-\frac{1}{4}\right)^{3}}{42}\left(\sin \left(\frac{1}{2}\right)-\sin \left(\frac{3}{4}\right)+\frac{1}{4} \cos \left(\frac{1}{4}\right)-\frac{3}{32} \sin \left(\frac{1}{4}\right)\right)+\left(1-\frac{\left(x-\frac{1}{4}\right)^{2}}{2}\right) \sin \left(\frac{1}{4}\right)+\left(x-\frac{1}{4}\right) \cos \left(\frac{1}{4}\right)+1
\end{array}\right) \\
-\frac{384}{7}\left(\begin{array}{l}
\left(x-\frac{1}{4}\right)^{3} \\
6
\end{array}-\left(x-\frac{1}{4}\right) \sin (x)\right)\left(\sin \left(\frac{1}{2}\right)-\sin \left(\frac{3}{4}\right)+\frac{1}{4} \cos \left(\frac{1}{4}\right)-\frac{3}{32} \sin \left(\frac{1}{4}\right)\right)+\left(x-\frac{1}{4}\right) \cos \left(\frac{1}{4}\right)
\end{array}\right) \\
+\left(\begin{array}{l}
\left.1+\sin (x)-\frac{\left(x-\frac{1}{4}\right)^{2}}{2}\right) \sin \left(\frac{1}{4}\right)-\sin (1+\sin (x))-(2+\sin (x)) \sin x=0
\end{array}\right) \\
\end{gathered}
$$

Nonlinear algebraic equation system achieved from Eq. (5.17) by using collocation points can be solved and the coefficients $C^{T}$ in Eq. (5.16) satisfied differential equation and whose boundary conditions are obtained. Table 4 shows the absolute errors for $M=4, k=0$, $M=4, k=1, M=4, k=2, M=8, k=0$ and $M=16, k=0$. As can be seen in Table 4 and Fig. 1, absolute errors tend to zero when number of grid points increase. The results obtained by the proposed method for small grid points such as $M=4, k=0$ are superior.

\section{Conclusion}

Chebyshev wavelet collocation method has been applied to the one linear and three nonlinear nonlocal boundary value problems. Approximate and exact solutions of examples are correspondingly compared. For Example 1, the comparisons of the absolute errors given in Table 1, it is clear that the results obtained by the proposed method are better than Reproducing Kernel Method [19]. Numerical results of Example 2 which is given in Table 2 are stable when number of grid points increase. It can be seen that results of proposed method for small grid points such as $M=4, k=0$ are superior to the results of Monotone Iterative Method [21] as given in Table 2. Numeric solutions of Example 3 for various values of $M$ and $k$ are given in Table 3. The precisions of approximate solutions obtained by the proposed method increase when number of grid points increase as can be seen in Table 3. For small grid points such as $M=4, k=0$, the results of proposed method are superior to the results of Homotopy Perturbation Method and Optimal Homotopy Asymptotic Method in [17]. Absolute errors of Example 4 are given in Table 4 and Fig 1 for various values of $M$ and $k$. As can be seen from Table 4 and Fig 1, absolute errors tend to zero when number of grid points increase and the proposed method is highly efficient and accurate. All of the calculations in this study have been made by the Maple program. Newton Raphson method has been used to solve nonlinear algebraic equation systems. These calculations demonstrate that the accuracy of the Chebyshev wavelet collocation method is quite good even for small number of grid points. In proposed method, there are no complex integrals or methodology. Applications of this method are very simple. It is also very convenient for solving the initial, boundary and nonlocal boundary value problems since the initial, boundary and nonlocal conditions are automatically taken in the solution. In addition, it can be concluded that the proposed method is reliable, simple, fast, minimal computation costs, flexible, and convenient alternative method. 
Figure 5.1: The absolute errors of Example 5.4 for various values of $M$ and $k$

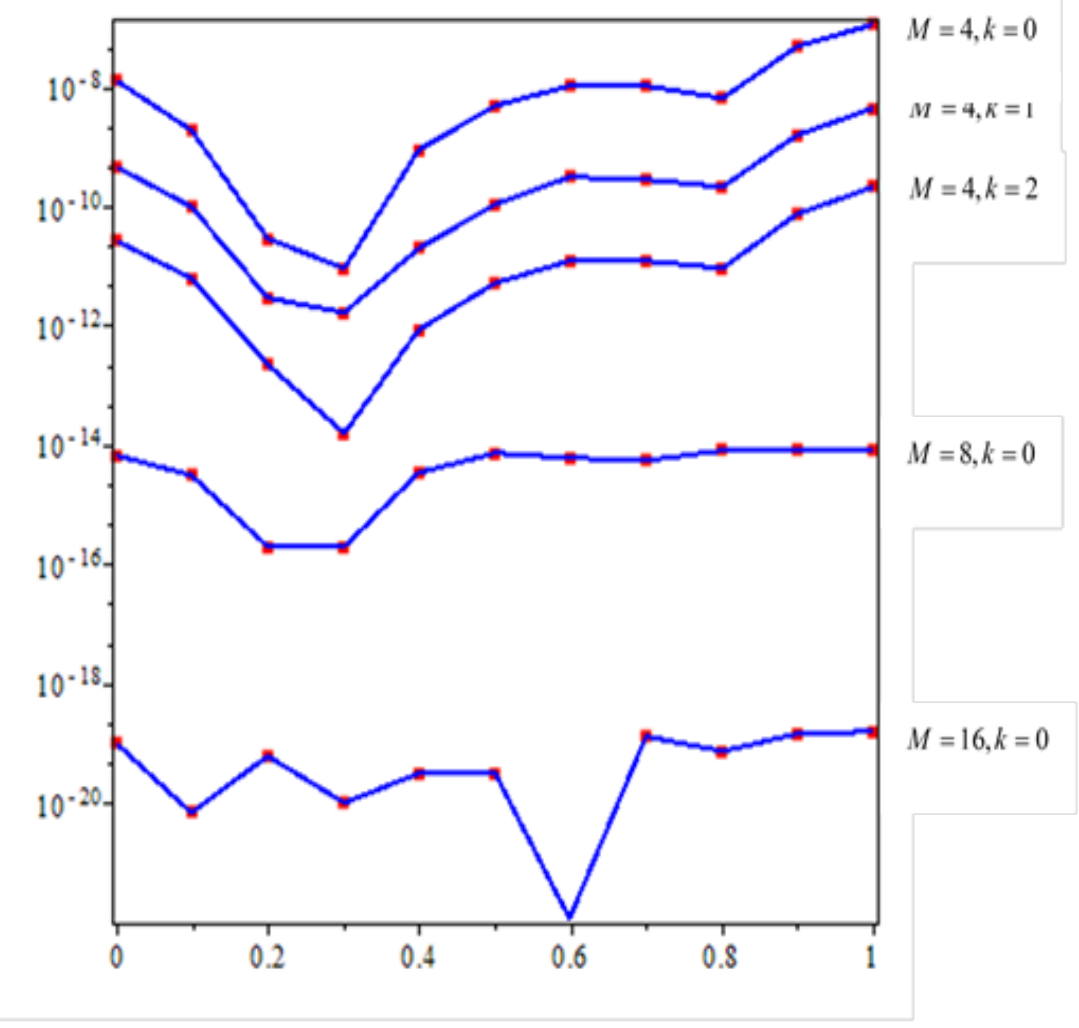

\section{References}

[1] A, Guezane-Lakoud, A. Frioui, Nonlinear three point boundary-value problem, Sarajevo Journal of Mathematics,8/20 (2012), $101-106$.

[2] B. Aksoylu, T. Mengesha, Results on nonlocal boundary value problems, Numerical functional analysis and optimization, 31/12 (2010), $1301-1317$.

[3] J. Henderson, CJ.Kunkel, Uniqueness of solution of linear nonlocal boundary value problems, Applied Mathematics Letters, 21 (2008), $1053-1056$.

[4] X. Xue, Nonlinear differential equations with nonlocal conditions in Banach spaces, Nonlinear Analysis: Theory, Methods and Applications , 63 (2005), $575-586$.

[5] P. Babak, Nonlocal initial problems for coupled reaction-diffusion systems and their applications, Nonlinear analysis: real world applications , 8 (2007), 980-996.

[6] J. Liang, TJ. Xiao, Semilinear integrodifferential equations with nonlocal initial conditions, Computers and Mathematics with Applications , 47 (2004), $863-875$.

[7] F. Geng, M. Cui, A repreducing kernel method for solving nonlocal fractional boundary value problems, Applied Mathematics Letters, (2012), 818-823.

[8] Y. Zhou, F. Jiao, Nonlocal Cauchy problem for fractional evolution equations, Nonlinear analysis: real world applications , 11 (2010), $4465-4475$.

[9] Lentini M, Pereyra V. A variable order ?nite difference method for nonlinear multipoint boundary value problems. Mathematics of Computation 1974; 28: $981-1003$

[10] MK. Kwong, The shooting method and multiple solutions of two/multi-point BVPs of second-order ODE, Electronic Journal of Qualitative Theory of Differential Equations, 6 (2006), 1-14.

[11] YK. Zou, QW. Hu and R. Zhang, On numerical studies of multi-point boundary value problem and its fold bifurcation, Applied Mathematics and Computation, 185 (2007), 527-537.

[12] M. Tatari, M. Dehghan, The use of the Adomian decomposition method for solving multipoint boundary value problems, Physical Scripta, 73 (2006), 672-676.

[13] M. Tatari, M. Dehghan, An ef?cient method for solving multi-point boundary value problems and applications in physics, Journal of Vibration and Control, (2011), 1116-1124.

[14] M. Dehghan, F. Shakeri, A semi-numerical technique for solving the multi-point boundary value problems and engineering applications, International Journal of Numerical Methods for Heat and Fluid Flow, 21 (2011), 794-809.

[15] A. Saadatmandi, M. Dehghan, The use of Sinc-collocation method for solving multi-point boundary value problems, Communications in Nonlinear Science and Numerical Simulation, 17 (2012), 593-601.

[16] LJ. Xie, CL. Zhou, S. Xu, A new algorithm based on differential transform method for solving multi-point boundary value problems, International Journal of Computer Mathematics (2015); 1-14

[17] J. Ali, S. Islam, S. Islam and G. Zaman, The solution of multipoint boundary value problems by the optimal homotopy asymptotic method, Computers and Mathematics with Applications, 59/6 (2010), 2000-2006.

[18] FZ. Geng, MG. Cui, Multi-point boundary value problem for optimal bridge design, International Journal of Computer Mathematics, 87 (2010), $1051-1056$.

[19] Y. Lin, J. Lin, A numerical algorithm for solving a class of linear nonlocal boundary value problems, Applied Mathematics Letters, 23 (2010), 997-1002.

[20] XY. Li, BY. Wu, Reproducing kernel method for singular fourth order four-point boundary value problems, Bulletin of the Malaysian Mathematical Sciences Society Second Series, 34/1 (2011), 147-151.

[21] B. Sun, Y. Aijun and G. Weigao, Successive iteration and positive solutions for some second-order three-point p-Laplacian boundary value problems, Mathematical and Computer Modelling, 50/3 (2009), 344-350.

[22] M. Behroozifar, Spectral method for solving high order nonlinear boundary value problems via operational matrices, BIT Numerical Mathematics, 55/4 (2015), 901-925

[23] D. Tzanetis, P. Vlamos, A nonlocal problem modelling ohmic heating with variable thermal conductivity, Nonlinear analysis: real world applications , 2/4 (2001), 443-454.

[24] M.Bogoya, A. Cesar and S. Gomez, On a nonlocal diffusion model with Neumann boundary conditions, Nonlinear Analysis: Theory, Methods and Applications, 75/6 (2012), 3198-3209.

[25] CV. Pao, Asymptotic behavior of solutions of reaction-diffusion equations with nonlocal boundary conditions, Journal of Computational and Applied Mathematics, 88 (1998), 225-238.

[26] CV. Pao, YM. Wang, Nonlinear fourth-order elliptic equations with nonlocal boundary conditions, Journal of Mathematical Analysis and Applications, 372 (2010), 351-365. 
[27] CV. Pao, YM. Wang, Numerical methods for fourth-order elliptic equations with nonlocal boundary conditions, Journal of Computational and Applied Mathematics, 292 (2016), 447-468.

[28] M. Razzaghi, S. Yousefi, Legendre wavelets direct method for variational problems, Mathematics and Computers in Simulation, 53 (2000) 185-192.

[29] M. Razzaghi, S. Yousefi, Legendre wavelets operational matrix of integration, International Journal of Systems Science , 32/4 (2001) 495-502.

[30] E, Babolian, F. Fattahzadeh, Numerical solution of differential equations by using Chebyshev wavelet operational matrix of integration, Applied Mathematics and Computation 188 (2007), 417-426

[31] MT. Kajania, AH. Vencheha and M.Ghasemib, The Chebyshev wavelets operational matrix of integration and product operation matrix, International Journal of Computer Mathematics, 86/7 (2009), 1118-1125

[32] İ. Çelik, Numerical Solution of Differential Equations by Using Chebyshev Wavelet Collocation Method, Cankaya University Journal of Science and Engineering., 10/2 (2013), 169-184.

[33] İ. Çelik, Chebyshev Wavelet Collocation Method for Solving Generalized Burgers-Huxley Equation, Mathematical Methods in the Applied Sciences, $39 / 3(2016), 366-377$

[34] I. Daubechies, Ten lectures on wavelets, SIAM, Philadelphia,PA (1992)

[35] L, Fox, IB. Parker, Chebyshev polynomials in numerical analysis, Oxford University Press, London (1968)

[36] H. Adibi, P. Assari, Chebyshev wavelet method for numerical solution of Fredholm integral equations of the first kind, Mathematical Problems in Engineering, Article ID 138408 (2010), p 17

[37] C. Yang, J.Hou, Chebyshev wavelets method for solving Bratu's problem, Boundary Value Problems, 2013 (2013), 142 